\title{
The expression of platelet-activating factor receptor modulates the cisplatin sensitivity of ovarian cancer cells: a novel target for combination therapy
}

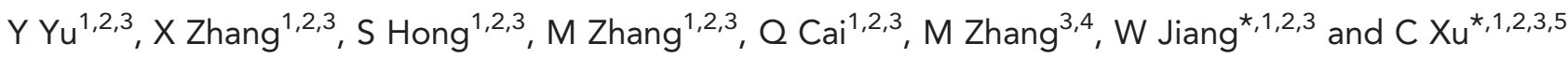 \\ ${ }^{1}$ Obstetrics and Gynecology Hospital, Fudan University, No.419, Fang-Xie Road, Shanghai 200011, China; ${ }^{2}$ Department of \\ Obstetrics and Gynecology of Shanghai Medical School, Fudan University, No.138, Yi-Xueyuan Road, Shanghai 200032, China; \\ ${ }^{3}$ Shanghai Key Laboratory of Female Reproductive Endocrine Related Diseases, No. 413, Zhao-Jiabang Road, Shanghai 200011, \\ China; ${ }^{4}$ Department of Obstetrics and Gynecology, Hospital 411 of CPLA, Shanghai 200081, China and ${ }^{5}$ Institute of Biomedical \\ Sciences, Fudan University, Shanghai 200032, No.138, Yi-Xueyuan Road, Shanghai 200032, China
}

Background: Ovarian cancer has the highest mortality rate of the gynaecological cancers. Although cisplatin (CDDP) is an effective treatment for ovarian cancer, recurrence is frequent and leads to death. The objective was to explore the role and possible mechanisms of platelet-activating factor receptor (PAFR) signalling in CDDP-treated ovarian cancer cells.

Methods: The upregulation of PAFR in CDDP-treated ovarian cancer cells was observed using realtime PCR and Western blot. The potential role of PAFR in modulating the CDDP sensitivity was assessed using a pharmacological inhibitor and siRNA knockdown. The PAFR-activated signalling pathways involved in cell responses to CDDP were assessed.

Results: Cisplatin induced increased PAFR expression in two ovarian cancer cell lines. The upregulation of PAFR by CDDP correlated with the time-dependent accumulation of NF- $\kappa \mathrm{B}$ and HIF-1 $\alpha$ in the nucleus. The inhibition of PAFR sensitised the ovarian cancer cells to CDDP. The PI3K and ERK pathways lie downstream of activated PAFR in CDDP-treated cells and their inhibition enhanced CDDP sensitivity. Finally, co-treatment with a PAFR antagonist (Ginkgolide B) and CDDP markedly reduced tumour growth in an in vivo model of ovarian cancer.

Conclusions: Together, these findings suggest that PAFR is a novel and promising therapeutic target for sensitising ovarian cancer cells to CDDP.

Ovarian cancer is the fifth most common cause of death from all cancers among women in the world and has the highest mortality rate of the gynaecological cancers (Chan and Mok, 2010). Overall, ovarian cancer has the worst prognosis of all gynaecological cancers, with a 5-year survival rate of $<40 \%$ (Baird et al, 2010). Surgical resection is the most effective and potentially curative therapy; however, most ovarian cancers are diagnosed at a late stage when the metastasis has already occurred (Bruchim et al,
2013). Under this scenario, chemotherapy is considered an option, but it provides only a modest improvement in patient survival (Rooth, 2013). Therefore, developing novel and effective therapeutic strategies against ovarian cancer is urgently needed.

Cisplatin (cis-diaminodichloroplatinum, CDDP) is a metalbased anti-cancer drug that has been used extensively over the past four decades for the treatment of several types of cancers (Shen et al, 2012), including ovarian cancer. However, most patients do 
not respond well to CDDP treatment and those who respond eventually develop chemoresistance and exhibit cancer progression (Laffman-Johnson, 2011). It is implied that cellular resistance to cisplatin is either inherent or acquired during the treatment; however, the underlying molecular mechanisms are still not wellestablished.

The role of platelet-activating factor (PAF)/platelet-activating factor receptor (PAFR) in tumours has been investigated in recent years. The PAF/PAFR axis is involved in oncogenic transformation (Kume and Shimizu, 1997), anti-apoptosis (Garcia et al, 2010), metastasis (Melnikova et al, 2009) and angiogenesis (Ferreira et al, 2007) in several types of cancers. We demonstrated that PAFPAFR is commonly activated in non-mucinous ovarian cancer cells (Aponte et al, 2008). A phospholipid mediator, PAF, can activate PAFR and promote ovarian cancer cell proliferation and invasion (Jiang et al, 2011). Recent experiments have also revealed an important role of PAF-PAFR in chemotherapy, demonstrating that PAFR activation can augment cytokine production induced by chemotherapy through a mechanism dependent on nuclear factorkappa B (NF- $\kappa$ B) (Darst et al, 2004). In this scenario, specific PAFR antagonists may have a potentiating effect in enhancing the CDDP sensitivity of ovarian cancer cells.

In this study, we examined the effect of cisplatin on PAFR expression in ovarian cancer cells to explore the possibility of a counter-defence mechanism. We demonstrated, for the first time, that cisplatin induces dose- and time-dependent upregulation of PAFR in ovarian cancer cells. The nuclear accumulation of NF- $\kappa \mathrm{B}$ and hypoxia inducible factor- 1 alpha $(\mathrm{HIF}-1 \alpha)$ is caused by CDDP, however, the silencing of these two transcription factors by RNA interference attenuates the CDDP-induced PAFR upregulation. We also showed that CDDP-induced PAFR expression induces the activation of the phosphoinositide 3-kinase (PI3K) and extracellular signal-regulated kinase (ERK) pathways; however, the simultaneous blockade of PI3K and ERK further sensitises ovarian cancer cells to CDDP. Furthermore, our data reveal that the PAFRspecific antagonist Ginkgolide B (GB) inhibits tumour growth in a SKOV-3-luc ovarian cancer model, reducing tumour progression when combined with cisplatin. Together, our results indicate that the expression of PAFR modulates the cisplatin sensitivity of ovarian cancer cells and is a novel target for combined therapy.

\section{MATERIALS AND METHODS}

Cell culture and chemical reagents. The ovarian cancer cell lines SKOV-3 and CAOV-3 (purchased from the Cell Bank of the Chinese Academy of Science, Shanghai, China) were cultured at $37^{\circ} \mathrm{C}$ in a humidified $5 \% \mathrm{CO}_{2}$ atmosphere in RPMI-1640 medium with $10 \%$ foetal calf serum (Gibco, Invitrogen, Carlsbad, CA, USA), $100 \mathrm{IU} \mathrm{ml}^{-1}$ penicillin $\mathrm{G}$, and $100 \mathrm{mg} \mathrm{ml}^{-1}$ streptomycin sulphate (Sigma-Aldrich, St Louis, MO, USA). Lipofectamine 2000 Transfection Reagent and Opti-MEM-1 Medium (Invitrogen) were used for siRNA transfection. The siRNA was synthesised by Shanghai GenePharma Co (Shanghai, China). Cis-diaminodichloroplatinum, WEB2086 (PAFR antagonist) and GB (>90\% high-performance liquid chromatography grade) were purchased from Sigma-Aldrich. PD98059 (ERK inhibitor) and LY294002 (PI3K inhibitor) were obtained from Cell Signaling Technology (Boston, MA, USA). A Cell Counting Kit8 (CCK-8) was purchased from Dojindo Molecular Technologies Inc. (Kumamoto, Japan) and the Alexa Fluor 488 annexin V/Dead Cell Apoptosis Kit was from Invitrogen. The rabbit polyclonal antibodies used in this study were directed against PAFR (Abcam, HongKong, China), NF- $\kappa$ B (Cell Signaling Technology), HIF-1 $\alpha$ (Cell Signaling Technology), Histone H3 (Cell Signaling Technology), phospho-P70S6K (Cell Signaling Technology), phospho-AKT and
phospho-ERK (Cell Signaling Technology). The mouse monoclonal antibodies used in this study were directed against actin and tubulin (Sigma, St Louis, MO, USA).

Quantitative real-time PCR. Real-time PCR analyses were performed with SYBR premix Ex Taq (TaKaRa, Osaka, Japan) on a 7900 Real-time PCR system (Applied Biosystems Inc., Foster, CA, USA). The primer sequences used for PAFR detection were as follows: sense- $5^{\prime}$-GACAGCATAGAGGCTGAGGC- ${ }^{\prime}$ and antisense- $5^{\prime}$-TAGCCATTAGCAATGACCCC- $3^{\prime}$. The $18 \mathrm{~s}$ rRNA used for normalisation was as follows: sense- $5^{\prime}$-GTAA CCCGTTGAACCCCATT- $3^{\prime}$ and antisense- $5^{\prime}$-CCATCCAATCGG TAGTAGCG- $3^{\prime}$. The $\Delta$ CT method was used to obtain the relative expression value of PAFR compared with the $18 \mathrm{~s}$ rRNA amplification.

Transfection of SiRNA. For the specific knockdown of PAFR, NF- $\kappa$ B and HIF- $1 \alpha$, cells were cultured in six-well plates and transiently transfected with $50 \mathrm{~nm}$ of non-target or target-specific siRNAs using Lipofectamine 2000 according to the manufacturer's instructions.

Nuclear and cytoplasmic fractionation. The preparation of the cytoplasmic and nuclear extracts was performed using the Nuclear Extract Kit (Active Motif, Carlsbad, CA, USA) as described previously.

Western blot analysis. Cellular extracts were prepared in a modified radioimmunoprecipitation assay buffer $(50 \mathrm{mM}$ Tris$\mathrm{HCl} \mathrm{pH} 7.4,1 \% \mathrm{NP}-40,0.25 \% \mathrm{Na}$-deoxycholate, $150 \mathrm{mM} \mathrm{NaCl}$, $1 \mathrm{~mm}$ EDTA, $1 \mathrm{~mm}$ PMSF, protease inhibitor cocktail). Protein concentrations of the cellular extracts were measured using a Bio-Rad protein assay kit (Hercules, CA, USA). Then, cellular extracts were subjected to SDS-PAGE. Proteins were transferred to PVDF membranes. After blocking for $1 \mathrm{~h}$ at room temperature in $5 \%$ BSA, blots were probed with a primary antibody at a $1: 1000$ dilution and incubated overnight at $4{ }^{\circ} \mathrm{C}$. Subsequently, blots were washed three times and incubated for $1 \mathrm{~h}$ at room temperature with a $1: 10000$ dilution of the secondary peroxidase-conjugated antibodies. Following three washes, immunoreactive bands were detected using electrochemiluminescence.

Cell proliferation assay and apoptosis assay. Cell proliferation was measured using a CCK-8 Assay, and cell apoptosis was detected using an Alexa Fluor 488 annexin V/Dead Cell Apoptosis Kit Assay. Both processes were conducted according to the manufacturer's protocols.

Immunofluorescence. The SKOV-3 cells with or without CDDP treatment were fixed with $100 \%$ methanol for $6 \mathrm{~min}$ at $-20^{\circ} \mathrm{C}$, then washed with PBS and left at $4{ }^{\circ} \mathrm{C}$ until use. Cells were permeabilized by incubation in PBS containing $0.3 \%$ Triton X-100 and $5 \%$ goat serum for $30 \mathrm{~min}$. A polyclonal antibody against NF- $\kappa \mathrm{B}$ and HIF- $1 \alpha$ were used at a $1: 100$ dilution, and a secondary antibody FITC-conjugated goat anti-rabbit (Invitrogen, was used at a $1: 200$ dilution. The first antibody was incubated overnight at $4{ }^{\circ} \mathrm{C}$ and the second antibody for $2 \mathrm{~h}$ at RT. Images were captured with an Olympus DP 71 camera (Tokyo, Japan). The magnification level was $400 \times$.

Animals and treatments. Female athymic nu/nu mice (4 to 6 weeks old) were obtained from the Laboratory Animal Center of the Shanghai Institutes for Biological Sciences of the Chinese Academy of Sciences. All animal studies were conducted strictly in accordance with protocols approved by the Ethics Committee for Animal Experimentation of Fudan University. To represent late stage ovarian cancer with abdominal metastasis, we used the SKOV-3-luc cells, which were transfected with a luciferaseexpressing vector kindly donated by $\mathrm{Dr} B$ in $\mathrm{Ye}$ from Harvard Medical School, Boston, MA, USA. We injected $2 \times 10^{6}$ 
SKOV-3-luc cells intraperitoneally (i.p.) into the female nu/nu mice. Luciferase activity was visualised through imaging of whole bodies using the in vivo imaging system Lumina (Caliper Life Sciences, Hopkinton, MA, USA) as described previously with minor adaptations at various time points post injection of cells. Briefly, animals were anaesthetised using the isoflurane-anesthesia system, d-luciferin dissolved in PBS (150 $\mathrm{mg} \mathrm{kg}^{-1}$; Caliper Life Science, Brussels, Belgium) was injected i.p. Animals were kept anaesthetised during the measurements, which were performed $5 \mathrm{~min}$ after the injection of D-luciferin. Bioluminescence imaging was acquired with a $10 \mathrm{~cm}$ field of view and an exposure time of $60 \mathrm{~s}$. The colour scale limits were set automatically and the quantitative analysis of bioluminescence was performed by measuring the luminescence signal intensity using the region of interest (ROI) settings of the Living Image 3.2 software (Caliper Life Sciences, Hopkinton, MA, USA). The measurements of ROI are expressed in total flux of photons. When the ROI of tumour tissues reached around $1 \times 10^{9}$, mice were randomly divided (10 mice per group) into the following four groups: DMSO vehicle control, GB-treated, CDDP-treated, or GB + CDDP-treated. The groups were treated with several different strategies. The GBtreated group was intraperitoneally injected with $100 \mu \mathrm{l}$ of $100 \mathrm{mg} \mathrm{ml}^{-1}$ of GB once per day for 3 weeks and the CDDPtreated group was intraperitoneally injected with $5 \mathrm{mg} \mathrm{kg}^{-1}$ of CDDP once per week for 3 weeks. The progress of tumour was visualised every 3 days by in vivo imaging system since first dosage of therapy. Statistical analysis was performed by Kruskal-Wallis and subsequent individual comparisons were performed by a multiple comparisons test using PASW Statistics 18 (SPSS Inc., Chicago, IL, USA). After 3 weeks of treatment, all mice were killed and the tumours were excised and weighed.

Immunohistochemistry (IHC). Immunohistochemical analysis was carried out for PAFR protein expression of ovarian cancer tissues in four treatment groups. Immunohistochemical analysis was performed using the EnVision/AP system (DakoCytomation, Carpinteria, CA, USA). This system used an anti-rabbit immunoglobulin conjugated to an alkaline phosphatase polymer (Labelled polymer-AP), which is revealed as a red stain after the addition of the Substrate-Chromogen solution. Slides were washed with deionized water and counterstained with hematoxylin and 5\% ammonium hydroxide and mounted in Accergel (Accurate Chemical and Scientific Corp., Westbury, NY, USA). The image was recorded by a digital camera (Optronic, Goleta, CA, USA). Each subject had three independent readings by two investigators using a semi-quantitative scoring system: 0 , absent or trace staining; 1, minimal staining; 2, moderate staining; or 3, strong staining.

Platelet-activating factor assay. Tail vein blood was collected from the nu/nu mice in each group at the different time points and the PAF concentrations were measured using a specific enzyme immunoassay kit (Groundwork Biotechnology Diagnosticate, San Diego, CA, USA). The assay was performed according to the manufacturer's instructions. Production of PAF was evaluated in duplicate, and amounts were taken from a standard curve of PAF. The sensitivity of the assay allowed the detection of up to $15 \mathrm{pg} \mathrm{ml}^{-1}$. When necessary, the samples were diluted in the assay buffer.

Statistical analysis. All experiments were performed at least three times. The data are expressed as the 'mean \pm s.d.'. Wherever appropriate, the data were also subjected to unpaired two-tailed Student's $t$-tests. Differences were considered significant when $P<0.05$.

\section{RESULTS}

Treatment with CDDP induces increased PAFR expression in ovarian cancer cells. The effect of CDDP on PAFR expression was examined in two human ovarian cancer cell lines, SKOV-3 and CAOV-3. After treatment with CDDP $(0-60 \mu \mathrm{M})$ for $24 \mathrm{~h}$, we observed a dose-dependent increase in the expression of PAFR mRNA and protein levels in both cell lines (Figure 1A and B, upper panel). A 1.2-fold upregulation of PAFR transcription was observed with the lowest dose of treatment, and the upregulation was more than 3.2-fold higher at the highest treatment dose. With increasing doses of CDDP, we also observed up to 3.4- and 4.0-fold increases in PAFR protein levels in SKOV-3 and CAOV-3 cells, respectively. In a time-gradient assay $(0-24 \mathrm{~h})$ with $10 \mu \mathrm{M}$ of CDDP, we observed an upregulation of the PAFR mRNA and protein levels in both cell lines as early as $1 \mathrm{~h}$ after treatment (Figure 1A and B, lower panel). Expression of PAFR increased with exposure time and reached maximum levels at $24 \mathrm{~h}$. These data show that the expression of PAFR in ovarian cancer cells is induced by exposure to the chemotherapeutic agent CDDP in a dose- and time-dependent manner.

Nuclear factor-kappa B and HIF-1 $\alpha$ are major players in the CDDP-induced expression of PAFR. Studies have reported that CDDP treatment modulates the activity of several transcription factors, including NF- $\kappa \mathrm{B}$ (Rohwer et al, 2010) and HIF- $1 \alpha$ (Ye et al, 2012). These transcription factors are also, reportedly, involved in the PAFR function in other cancer types (Keely et al, 2010). To investigate whether NF- $\kappa$ B and HIF- $1 \alpha$ mediate CDDPinduced upregulation of PAFR, we observed the change in their expression and subcellular localisation following CDDP treatment. The results demonstrate that the CDDP treatment of SKOV-3 and CAOV-3 cells causes a time-dependent increase in the nuclear levels of NF- $\kappa \mathrm{B} / \mathrm{p} 65$ (Figure $2 \mathrm{~A}$ and B, upper panel) with a corresponding decrease in the cytoplasmic levels (Figure 2A and $\mathrm{B}$, middle panel). Furthermore, after CDDP treatment, we also detected an increase in nuclear levels of HIF- $1 \alpha$ (Figure 2A and B, upper panel) as well as an increase in the cytoplamic fractions of ovarian cancer cell lysates (Figure 2A and B, middle panel). However, we did not observe a change in total NF- $\kappa \mathrm{B}$ and HIF- $1 \alpha$ levels in whole cell lysates (Figure 2A and B, lower panel). As shown in Figure 2C, immunofluorescence localisation of NF- $\kappa$ B and HIF- $1 \alpha$ in SKOV- 3 cells further confirmed that CDDP treatment induces nuclear accumulation of NF- $\kappa \mathrm{B}$ and HIF- $1 \alpha$. We next investigated the role of NF- $\kappa$ B and HIF- $1 \alpha$ in the CDDP-induced upregulation of PAFR. Using RNA interference, we silenced the expression of NF- $\kappa \mathrm{B}$ and HIF- $1 \alpha$ in the SKOV-3 and CAOV-3 cells prior to treatment with CDDP. Our results show that siRNAs targeting NF- $\kappa$ B and HIF- $1 \alpha$ were able to effectively silence the expression of these transcription factors, leading to their reduced nuclear accumulation. In addition, our data show that suppression of NF- $\kappa \mathrm{B}$ or HIF- $1 \alpha$ results in the abrogation of CDDP-induced PAFR expression, although the effect of NF- $\kappa \mathrm{B}$ silencing on PAFR expression (Figure $3 \mathrm{~A}$ and $\mathrm{B}$ ) is more potent compared with HIF-1 $\alpha$ silencing (Figure $3 \mathrm{C}$ and D). To further analyse the effect of NF- $\kappa$ B and HIF- $1 \alpha$ inhibition on CDDP-induced apoptosis, we performed Annexin V/PI analysis (Figure $3 \mathrm{E}$ and $\mathrm{F}$ ), our data show that the suppression of NF- $\kappa \mathrm{B}$ and HIF- $1 \alpha$ led to an increase in cell apoptosis when cells were exposed to CDDP compared with CDDP alone. Together, these data demonstrate the role of NF- $\kappa \mathrm{B}$ and HIF- $1 \alpha$ in CDDP-induced PAFR upregulation.

Suppression of PAFR with WEB2086 or depletion after siRNA silencing significantly enhances the CDDP sensitivity of ovarian cancer cells. To investigate the role of upregulated PAFR in the response of ovarian cancer cells to CDDP, SKOV-3 and CAOV-3 cells were pretreated with the PAFR small-molecular antagonist WEB2086 before exposure to CDDP $(10 \mu \mathrm{M})$. As shown in Figure 4A, PAFR inhibition by WEB2086 enhanced cell death in both ovarian cancer cell lines after CDDP treatment. When PAF was added to the cell culture, there was a significant decrease in cell death, however, there was a clear increase in cell death when cells 
A

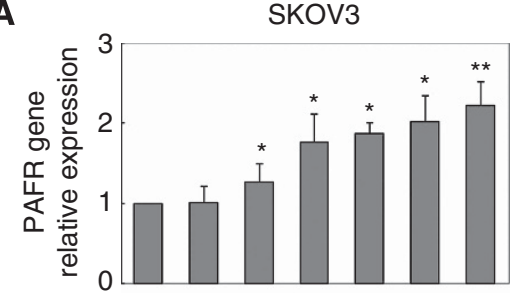

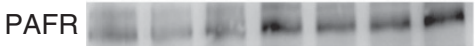

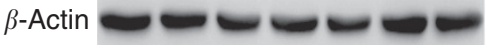

$\operatorname{CDDP}(\mu \mathrm{M}) \quad 0 \quad 2.5 \quad 5 \quad 10 \quad 20 \quad 40 \quad 60$

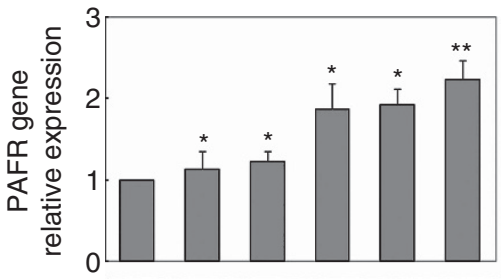

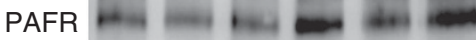

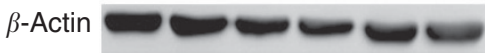

B
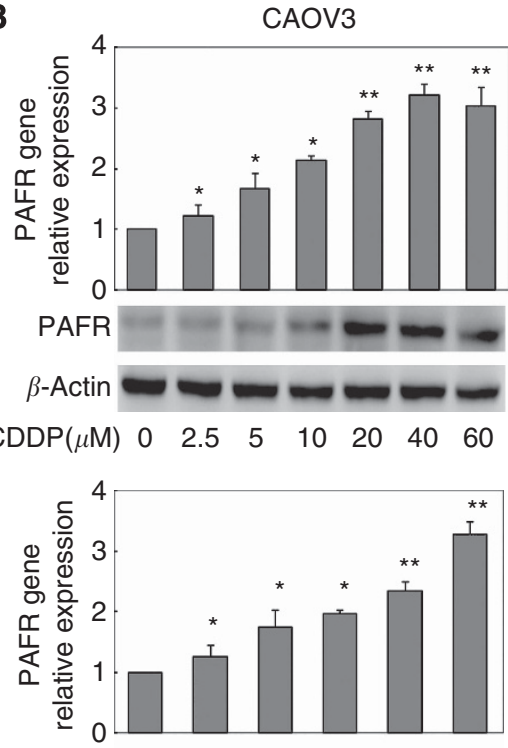

PAFR $\rightarrow=-m=0$

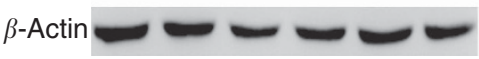

Figure 1. Cisplatin induces increased PAFR expression in human ovarian cancer cells. Ovarian cancer cells were treated with different doses $(0-60 \mu \mathrm{M})$ of cisplatin (CDDP) for $24 \mathrm{~h}$ (A and B, upper panels) or with a constant dose (10 $\mu \mathrm{M})$ of CDDP for different time intervals as indicated (A and B, lower panels). Total RNA and protein were extracted and analysed for PAFR expression by real-time PCR and immunoblot analysis, respectively. $18 \mathrm{~s}$ rRNA (realtime PCR) and $\beta$-actin (immunoblot) were used as internal controls. The data show that CDDP induces a doseand time-dependent expression of PAFR levels as a transcript and as a protein. Bars represent the average of the triplicates \pm s.d.; ${ }^{*}(P<0.05)$ and ${ }^{* *}(P<0.01)$ indicate a statistically significant difference compared with the untreated control.

A
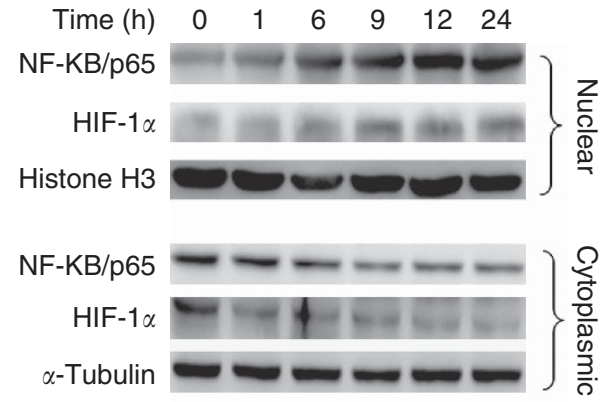

NF-KB/p65 $=-\longrightarrow$

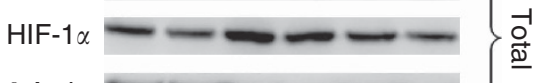

$\beta$-Actin

C

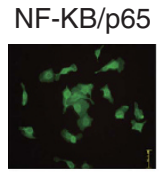

DAPI

Merge
Untreated
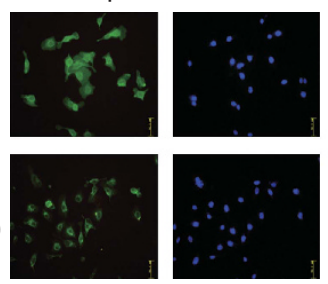

CDDP

$(10 \mu \mathrm{M} 12 \mathrm{~h})$

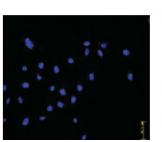

B

Time (h) $\quad 0 \quad 1 \quad 6 \quad 9 \quad 12 \quad 24$

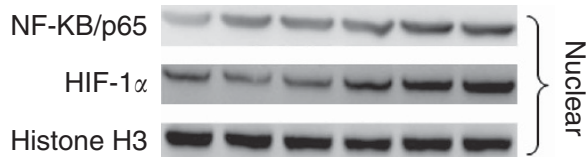

NF-KB/p65

HIF-1 $\alpha$

$\alpha-$ Tubulin

NF-KB/p65

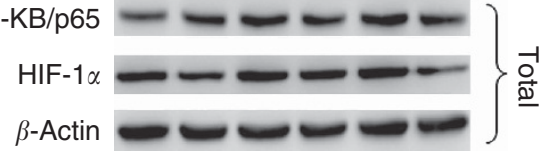

$\mathrm{HIF}-1 \alpha$

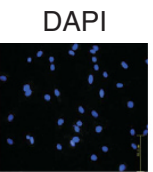

Merge

Untreated
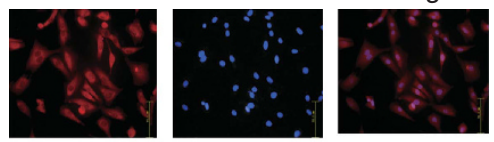

CDDP

$(10 \mu \mathrm{M} 12 \mathrm{~h})$
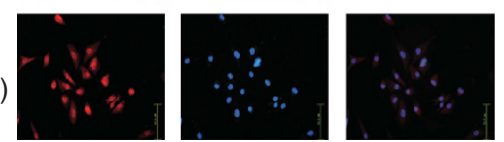

Figure 2. Treatment with CDDP enhances the nuclear localisation of NF- $\kappa \mathrm{B} / \mathrm{p} 65$ and HIF- $1 \alpha$. The SKOV-3 (A) and CAOV-3 (B) cells were treated with CDDP $(10 \mu \mathrm{m})$ at different time intervals $(0-24 \mathrm{~h})$. Thereafter, nuclear, cytoplasmic and total extracts were prepared and the expression and localisation of NF- $\kappa \mathrm{B} / \mathrm{p} 65$ and HIF-1 $\alpha$ were examined by immunoblot analysis. Histone $\mathrm{H} 3$ (for the nuclear fraction), $\alpha$-tubulin (for the cytoplasmic fraction) and $\beta$-actin (for the total fraction) were used as loading controls. (C) The localisation of NF- $\kappa \mathrm{B} / \mathrm{p} 65$ and HIF- $1 \alpha$ in SKOV-3 cells with or without $10 \mu \mathrm{M}$ CDDP for $12 \mathrm{~h}$ treatment were analysed using immunofluorescence. The data indicate increased levels of NF- $\kappa \mathrm{B} / \mathrm{p} 65$ and HIF-1 $\alpha$ in the nuclei of the ovarian cancer cells treated with CDDP. 
A
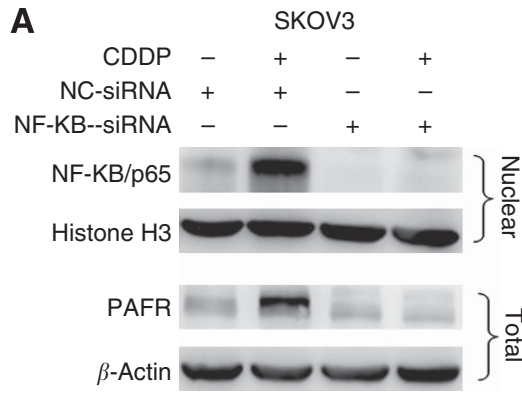

C
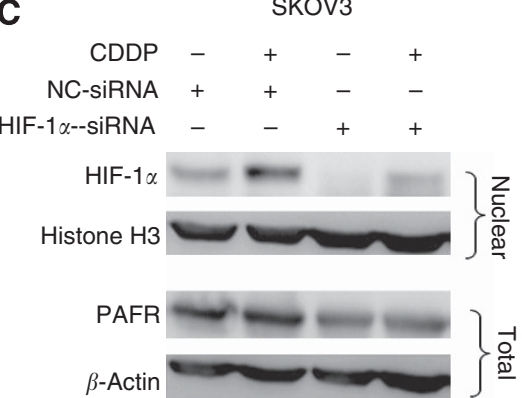

E

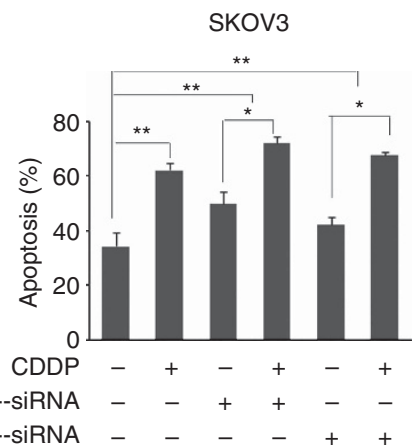

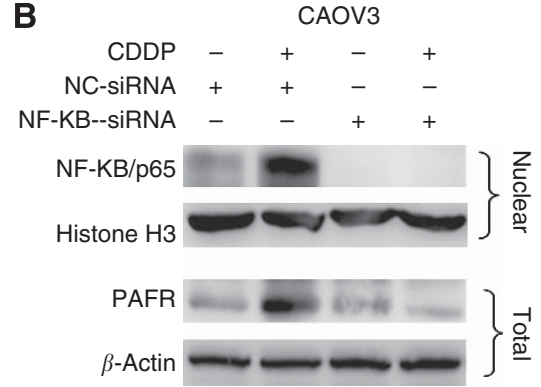

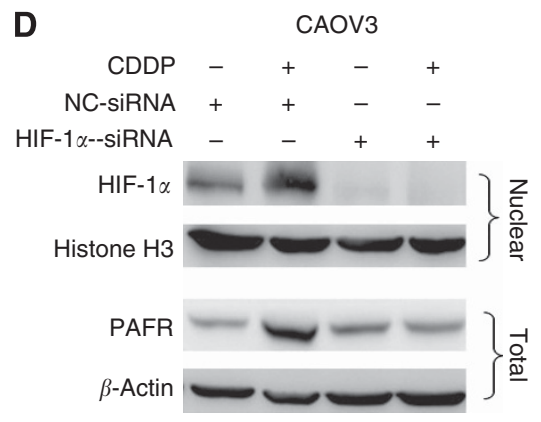

$\mathbf{F}$

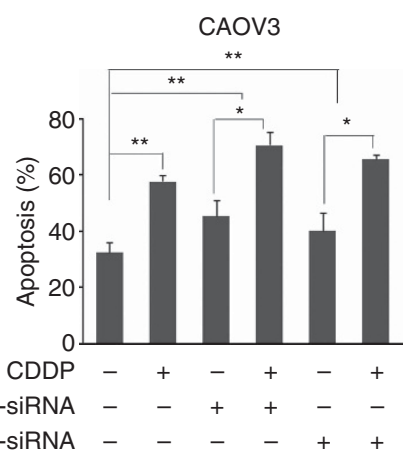

Figure 3. The involvement of NF- $\kappa \mathrm{B} / \mathrm{p} 65$ and HIF-1 $\alpha$ in CDDP-induced PAFR expression in ovarian cancer cells. Sub-confluent ovarian cancer cells were transiently transfected with a negative-control (NC) or siRNAs targeting either NF- $\kappa$ B/p65 (A and B) or HIF-1 $\alpha$ (C and D). Following $48 \mathrm{~h}$ of transfection, the NF- $k \mathrm{~B} / \mathrm{p} 65$ and HIF- $1 \alpha$ cells were treated with CDDP $(10 \mu \mathrm{m})$ for the next $12 \mathrm{~h}$, and the PAFR cells were treated with CDDP $(10 \mu \mathrm{M})$ for the next $24 \mathrm{~h}$. Nuclear and total proteins were isolated and subjected to immunoblot analysis to assess the expression of NF- $\kappa \mathrm{B} / \mathrm{p} 65$, HIF- $1 \alpha$ and PAFR. Histone $\mathrm{H} 3$ and $\beta$-actin were used as loading controls for the nuclear and total proteins, respectively. (E and F) Following $48 \mathrm{~h}$ of transfection, cells were treated with CDDP $(10 \mu \mathrm{m})$ for the next $12 \mathrm{~h}$. Cell apoptosis was assessed by flow cytometric analysis. The data indicate that specific silencing of NF- $\kappa \mathrm{B} / \mathrm{p} 65$ and HIF-1 $\alpha$ enhance the sensitivity of CDDP and clearly demonstrate that CDDP-induced PAFR expression is mediated through the additive action of NF- $\kappa \mathrm{B} / \mathrm{p} 65$ and HIF-1 $\alpha$ in ovarian cancer cells.

were exposed to CDDP and WEB2086, compared with CDDP alone. To further analyse the effect of PAFR inhibition on CDDPinduced apoptosis, we performed an Annexin V/PI analysis (Figure 4B). The results show that there was an increase in cell apoptosis when the cells were exposed to CDDP and WEB2086 compared with CDDP alone. Furthermore, through RNA interference, we suppressed the expression of PAFR in SKOV-3 and CAOV -3 cells before treatment with CDDP. Our data indicate that PAFR-targeted siRNAs were able to effectively silence PAFR expression and that the suppression of PAFR led to an increase in cell apoptosis when cells were exposed to CDDP compared with CDDP alone (Figure 4C). As shown in Figure 4C, the apoptosis results were confirmed by a Western blot analysis. Both PAFRtargeted siRNA and CDDP were able to induce the cleavage of 113$\mathrm{kDa}$ poly-ADP-ribose polymerase (PARP) to the $89-\mathrm{kDa}$ fragment and induce cleaved caspase- 3 in SKOV-3 and CAOV-3 cells, and the combination of two agents was accompanied by increased expression of cleaved PARP and caspase-3. Together, our data show that suppression of PAFR significantly enhanced the CDDP sensitivity of ovarian cancer cells.
Phosphoinositide 3-kinase and ERK lie downstream from activated PAFR in CDDP-treated ovarian cancer cells. We next investigated the downstream signalling pathway of activated PAFR in CDDP-treated ovarian cancer cells. The activation of the PI3K and ERK signal pathways is a common cause of cell apoptosis or survival (Yu and Kim, 2013). Additionally, the PAF-PAFR signalling axis has been shown to activate AKT and ERK in several types of cancer cells (Aponte et al, 2008; Jiang et al, 2011). Therefore, we next examined whether PI3K and ERK might function downstream of PAFR in CDDP-treated ovarian cancer cells. In a time-course assay $(0-24 \mathrm{~h})$ with a $10 \mu \mathrm{M}$ CDDP treatment, we observed maximum activation of P70S6K/Akt/Erk phosphorylation at $12 \mathrm{~h}$ in the SOKV-3 cells and at $6 \mathrm{~h}$ in the CAOV-3 cells (Figure 5A and C). Furthermore, when PAF was added to the cell culture, there was a marked increase in the amount of P70S6K/Akt/Erk phosphorylation in both cells (Figure $5 \mathrm{~A}$ and $\mathrm{C}$ ). To investigate whether the activation of the PI3K and ERK pathways is PAFR-dependent, WEB2086 was added to the culture medium $1 \mathrm{~h}$ before CDDP and/or PAF stimulation. In both the SKOV-3 and CAOV-3 cells, we observed a decrease in 
A

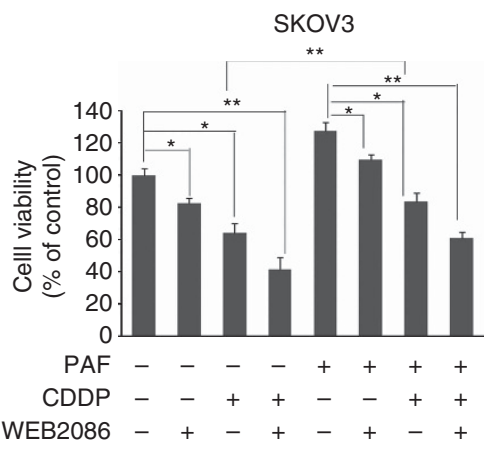

B

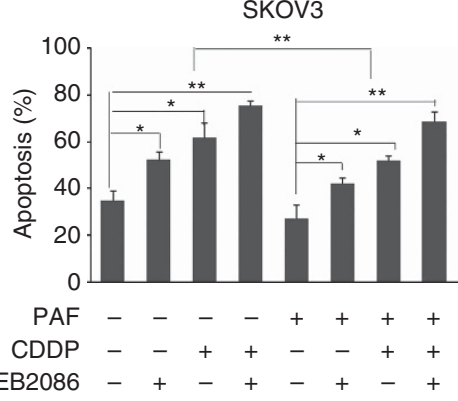

C
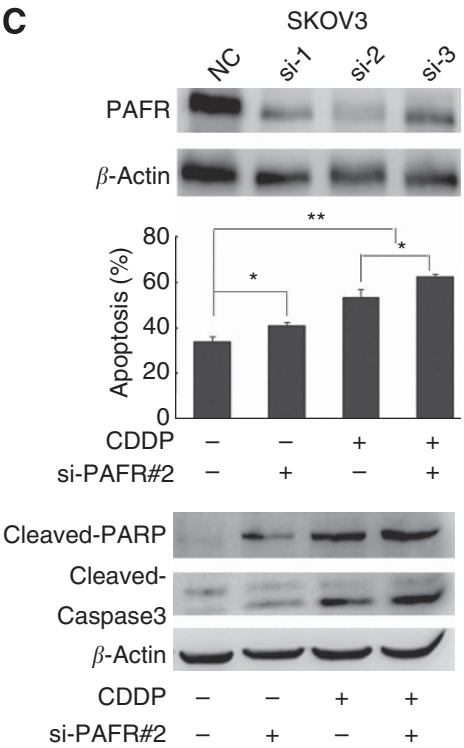

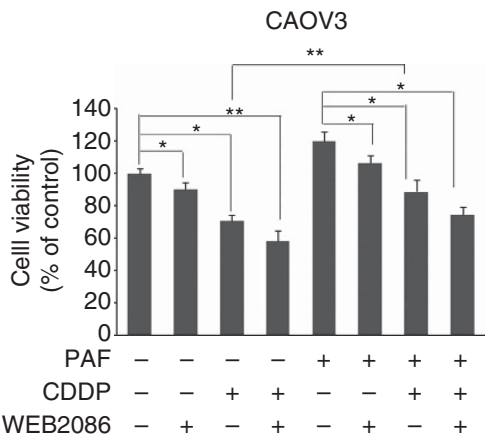

CAOV3

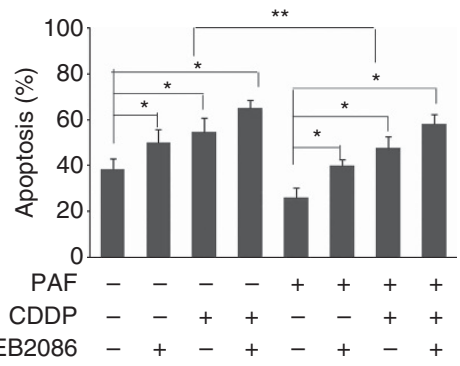

CAOV3
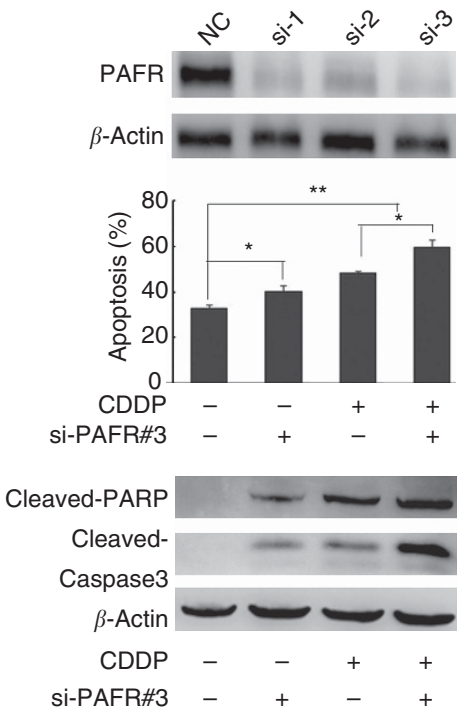

Figure 4. PAFR suppression with a pharmacological inhibitor or siRNA knockdown enhances the CDDP sensitivity of ovarian cancer cells. The SKOV-3 and CAOV-3 cells were pretreated with the PAFR-antagonist WEB2086 (50 or $100 \mu \mathrm{M})$ and then treated with CDDP (10 $\mu \mathrm{m})$ for $24 \mathrm{~h}$ in the presence or absence of PAF. Cell viability was assayed using a CCK-8 assay (A), and cell apoptosis was determined by AnnexinV/PI flow cytometric analysis (B). Both cell lines were transfected with a negative-control (NC) or PAFR-targeted siRNAs. Following $48 \mathrm{~h}$ of transfection, cells were treated with CDDP $(10 \mu \mathrm{M})$ for $24 \mathrm{~h}$. Cell apoptosis was assessed by flow cytometric analysis (C). Western blotting analysis of PARP and caspase-3 cleavage following treatment with PAFR-targeted siRNA or with CDDP in both cell lines (C). Western blotting of $\beta$-actin is included as a loading control. The data indicate that WEB2086 and si-PAFR significantly enhance the CDDP sensitivity of ovarian cancer cells. Bars represent the average of the triplicates \pm s.d.; * $(P<0.05)$ and ${ }^{* *}(P<0.01)$ indicate a statistically significant difference compared with the untreated control.

The data are representative of three independent experiments.

the phosphorylation of P70S6K/Akt/Erk as we increased the concentration of WEB2086 $(25-100 \mu \mathrm{M})$ (Figure 5B and D). Together, our data suggest that PI3K and ERK activation occurs downstream of the PAF-PAFR signalling axis in CDDP-treated ovarian cancer cells.

The blockade of the PI3K and ERK pathways sensitises ovarian cancer cells to CDDP. To evaluate whether PAFR-dependent $\mathrm{PI} 3 \mathrm{~K}$ and ERK activation is involved in the modulation of the ovarian cancer cell responses to CDDP, we treated the SKOV-3 and CAOV-3 cells with specific pharmacological inhibitors of PI3K (LY294002) and ERK (PD98059) prior to stimulation with PAF and CDDP. Immunoblot analyses demonstrated effective and specific inhibition of activated PAFR-induced phosphorylation of P70S6K/Akt/Erk in both of the CDDP-treated cell lines (Figure 6A). The inhibition of growth and the proportion of apoptosis were evaluated by a CCK-8 assay (Figure 6B) and a flow cytometry assay (Figure 6C), respectively. Both the inhibition of growth and the proportion of apoptosis were significantly higher in the cells treated with a co-administration of PAF, CDDP, PD98059 
A

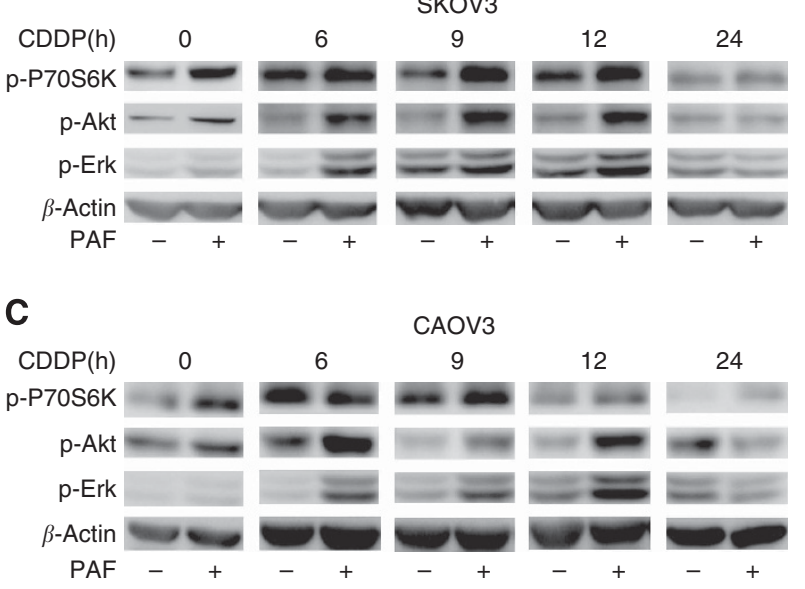

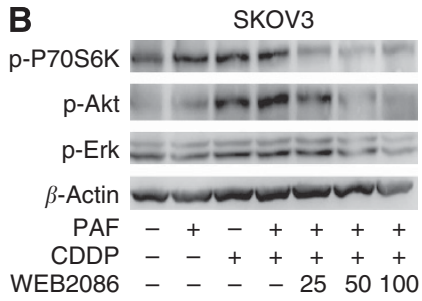

D

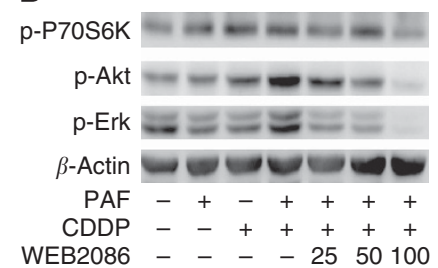

Figure 5. The PI3K and ERK signalling lies downstream of activated PAFR in CDDP-treated ovarian cancer cells. SKOV-3 (A) and CAOV-3 (C) cells were treated with CDDP $(10 \mu \mathrm{M})$ at different time intervals $(0-24 \mathrm{~h})$. Meanwhile, CDDP-treated cells at every point in time were stimulated with or without PAF (100 nM). Thereafter, total extracts were prepared and the phosphorylation of P70S6K/Akt/Erk was examined by immunoblot analysis. Both cell lines were pretreated with different doses of PAFR-antagonist WEB2086 (25-100 $\mu \mathrm{m})$ for $1 \mathrm{~h}$ before stimulation with PAF (100 nM) and CDDP $(10 \mu \mathrm{M})$ for $24 \mathrm{~h}$ (B and D). Total proteins were extracted, and the phosphorylation of P70S6K/Akt/Erk was examined by immunoblot analysis. $\beta$-actin was used as a loading control. The data show that $\mathrm{p}$-PI3K/Akt/Erk was activated by PAFR in CDDP-treated cells and that the activation was PAFR-dependent. There was no different expression of total-P70S6K, total-Akt and total-Erk in each group (data not shown).

and LY294002 compared with the cells treated with PAF-CDDP alone or PAF-CDDP plus either agent. Meanwhile, treatment with PD98059 or LY294002 alone only slightly affected cell viability and apoptosis in the SKOV-3 and CAOV-3 cells. Together, these data indicate that the PI3K and ERK pathways downstream of activated PAFR are associated with CDDP responses in ovarian cancer cells.

Inhibition of PAFR significantly sensitises CDDP therapy in an ovarian cancer model. To bolster our in vitro findings and to determine whether PAFR could be an effective therapeutic target for ovarian cancer, we examined the effects of GB in an ovarian cancer animal model by intraperitoneally implanting SKOV-3-luc cells into mice. Ginkgolide B is the primary active component of Ginkgo biloba extracts and has proven to be an effective antagonist against PAFR. The fluorescence intensity (ROI) values represented the tumour volume in each group, and the ROI curves showed a slight decrease in tumour volume in mice treated with CDDP or $\mathrm{GB}$ in comparison with the control group. However, there was significant inhibition of tumour growth in the group subjected to the combination therapy (CDDP and GB) (Figure 7A and B). This group also showed the lightest tumour tissues (Figure $7 \mathrm{C}$ ). Furthermore, to detect the concentration of PAF, we collected the tail vein blood of the nu/nu mice in each group at the following time points: before implanting the SKOV-3-luc cells, before the therapy treatment and 2 weeks after the treatment. As shown in Figure $7 \mathrm{D}$, the serum PAF values were elevated after the i.p. ovarian cancer cells injection but were not altered by the other treatments in all four groups. Immunohistochemical staining on the ovarian cancer tissues of four treatment groups revealed that PAFR protein expression was significantly increased after 3 weeks of CDDP treatment. The inhibition of PAFR expression was observed when the combination treatment with GB and CDDP was executed (Figure 7E). Together, these data suggest that the combination therapy with GB and CDDP was effective in reducing tumour progression.

\section{DISCUSSION}

The PAF/PAFR signalling axis has emerged as an important determinant of aggressive phenotypes in several malignancies
(Zhang et al, 2010; Sahu et al, 2012). Our earlier data demonstrated that GB, a specific antagonist of PAFR, had an additive effect with the most commonly used chemotherapy agent, CDDP, and exhibited significantly less cytotoxicity than CDDP (Jiang et al, 2012). The present study provides additional support for the functional significance of the PAF/PAFR signalling axis as a resistance mechanism by demonstrating an upregulation of PAFR in response to CDDP treatment of ovarian cancer cells. Transcriptional upregulation of PAFR is facilitated by the nuclear accumulation of the NF- $\kappa \mathrm{B}$ and HIF- $1 \alpha$ transcription factors. Selective PAFR suppression with WEB2086 or RNA interferencemediated knockdown results in the enhancement of CDDP sensitivity of the ovarian cancer cells. In addition, we found that downstream of PAF/PAFR, the PI3K and ERK signalling pathways are two of the potential mechanisms for the regulation of CDDP responses. Finally, our in vivo ovarian cancer model results showed that, in the presence of CDDP, the PAFR-specific antagonist GB, inhibited tumour growth, thus demonstrating the therapeutic effect of PAFR suppression. Together, our findings suggest that CDDPinduced PAFR upregulation modulates the CDDP sensitivity of ovarian cancer cells.

Expression of PAFR is elevated in non-mucinous types of ovarian cancer tissues and cells, which suggests that it has a role in the pathogenesis and progression of ovarian cancer. The sole ligand of PAFR, PAF, is secreted by many different cell types, including endothelial, stromal and inflammatory cells. The PAF is also secreted by many different tumour cells (Li et al, 2003; Braeuer et al, 2011), thus indicating an important role for PAF/PAFR signalling in ovarian cancer progression. In addition, it has been shown that PAF/PAFR significantly promotes ovarian cancer proliferation and invasion. In the present study, we observed that CDDP treatment led to a dose- and time-dependent increase in PAFR expression. This observation provides additional support for our earlier findings, which showed that, GB may sensitise ovarian cancer cells to cisplatin. Our data suggest that induction of PAFR upon CDDP treatment could be a counter defense mechanism of ovarian cancer cell resistance to apoptosis by promotion of PAF/PAFR-induced cell survival pathways.

Our data show direct roles of NF- $\kappa \mathrm{B} / \mathrm{p} 65$ and HIF- $1 \alpha$ in the CDDP-induced upregulation of PAFR expression in ovarian cancer cells. Activation of NF- $\kappa \mathrm{B} / \mathrm{p} 65$ and HIF- $1 \alpha$ transcription activity 
A

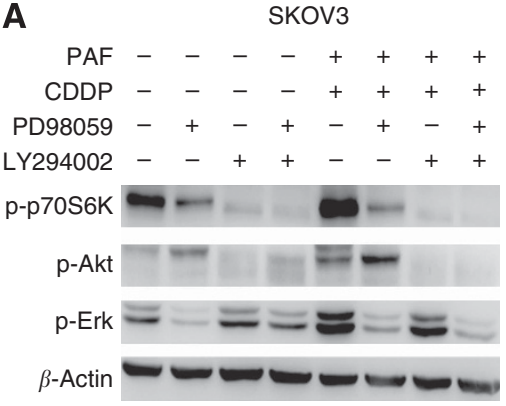

B
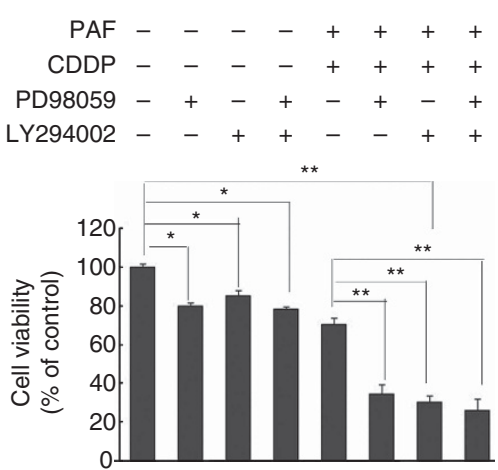

C

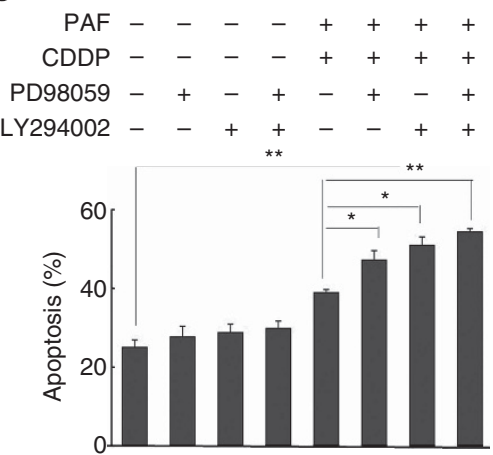

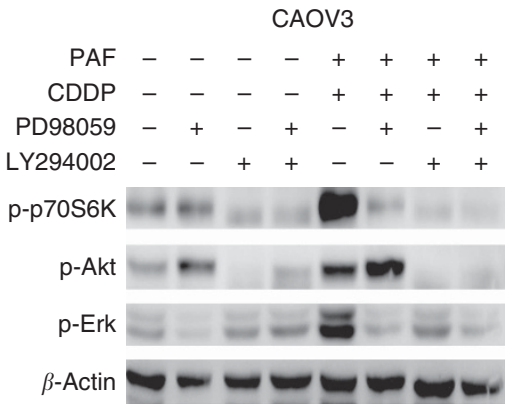

CAOV3

\begin{tabular}{|c|c|c|c|c|c|c|c|}
\hline PAF & - & - & - & - & + & + & + \\
\hline CDDP & - & - & - & - & + & + & + \\
\hline PD98059 & - & + & - & + & - & + & - \\
\hline LY294002 & - & - & + & + & - & - & + \\
\hline
\end{tabular}

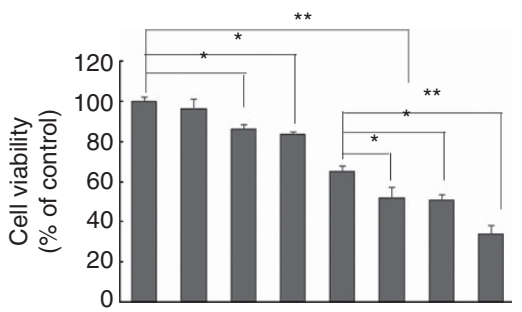

CAOV3

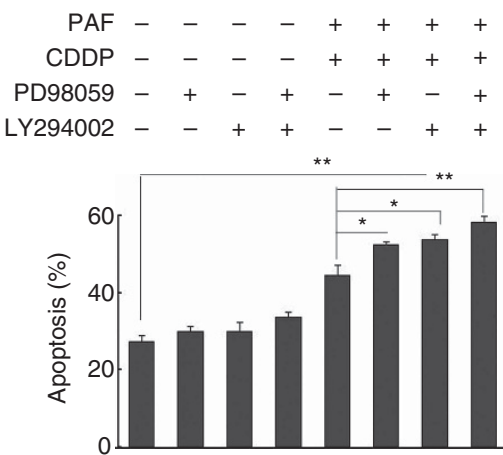

Figure 6. The effect of the PI3K and ERK inhibitors on cell viability and apoptosis in CDDP-treated and PAFR-activated ovarian cancer cells. (A) SKOV-3 and CAOV-3 cells were pretreated with PD98059 $(20 \mu \mathrm{M})$ and/or LY294002 (20 $\mu \mathrm{M})$ for $1 \mathrm{~h}$ before exposure to PAF (100 nM) and CDDP $(10 \mu \mathrm{M})$ for $24 \mathrm{~h}$. Total protein was extracted and analysed for phosphorylation and P70S6K/Akt/Erk expression by immunoblot analysis. $\beta$-actin was used in the immunoblot as an internal control. (B) SKOV-3 and CAOV-3 cells were pretreated with PD98059 and IY294002 before PAF and CDDP treatment for $24 \mathrm{~h}$. Viable cells were detected using a CCK-8 assay. (C) The proportion of cell apoptosis in both cell lines was assessed by flow cytometric analysis after treatment for $24 \mathrm{~h}$ with the indicated reagents. Bars represent the means \pm s.d. $;^{*}(P<0.05)$ and $* *(P<0.01)$ indicate a statistically significant difference compared with the untreated control. The data are representative of three independent experiments. There was no different expression of total-P70S6K, total-Akt and total-Erk in each group (data not shown).

by chemotherapy agents has previously been reported and has been shown to be essential for oncogenic transformation (Liu et al, 2010; Singh et al, 2012). Our data reveal that the effect of NF- $\kappa \mathrm{B}$ silencing on PAFR expression is more potent compared with HIF- $1 \alpha$ silencing, suggesting that there is a possibility that CDDP-induced HIF- $1 \alpha$ upregulation is NF- $\kappa \mathrm{B} / \mathrm{p} 65$ dependent. This finding is supported by published data on other cancers in which NF- $\kappa$ B and HIF- $1 \alpha$ have had roles in CXCR4 (a G-protein coupled receptor) expression (Oh et al, 2012). However, as transcription factors, NF- $\kappa \mathrm{B}$ and HIF- $1 \alpha$ can interact with a variety of proteins; thus, we need to further analyse the mechanism behind the CDDP induction of increased PAFR expression.

The PAF-PAFR-mediated stimulation of the downstream phosphorylation of multiple proteins has been reported in other non-cancerous types of cells (Mollapour et al, 2001) and involve many types of protein kinases including mitogen-activated protein kinase, protein kinase $\mathrm{C}$ and $\mathrm{PI} 3 \mathrm{~K}$, among others (Ishii and Shimizu, 2000). Our previous study demonstrated that PAF/PAFR, through FAK, PI3K and MMP2, initiates multiple downstream signal pathways involved in ovarian cancer cell proliferation and invasion. In addition, the PI3K and ERK pathways, which mediate critical signals for cell proliferation, survival and differentiation (Siddiqa et al, 2008), are activated in various types of cancer. Several other studies have also shown that the constitutive or induced activation of the PI3K and ERK pathways has been associated with the chemoresistance of cancer cells (Yoon et al, 2011; Gao et al, 2013). In the present study, our data show that as CDDP induced the upregulation of PAFR, there was a concomitant 
A

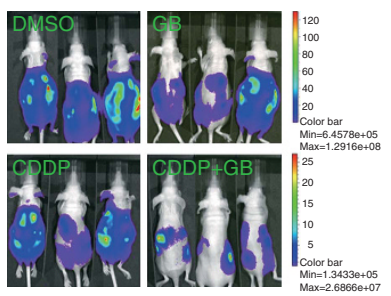

B

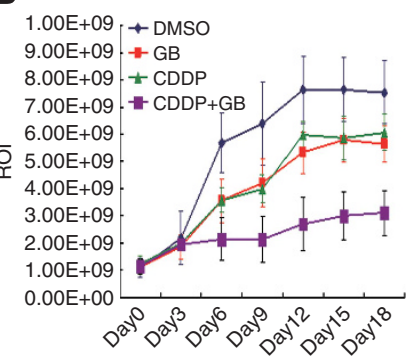

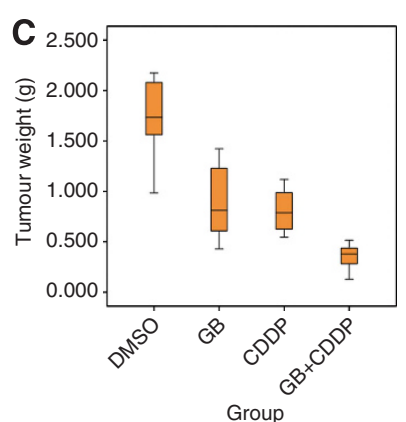

E
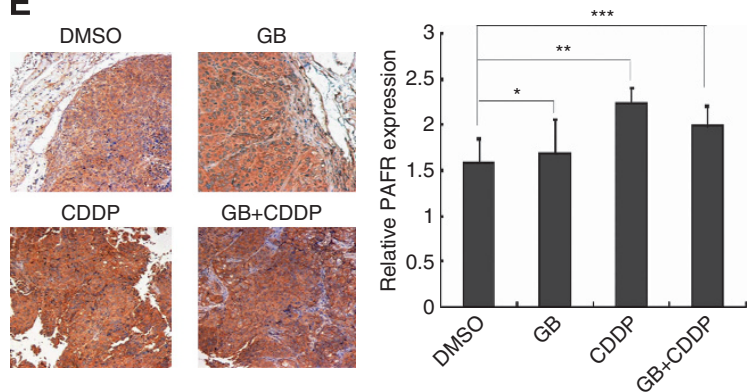

Figure 7. Inhibition of PAFR significantly sensitises CDDP therapy in an ovarian cancer model. (A) The representative in vivo imaging picture of the four groups of nude mice with peritoneal ovarian cancer. SKOV-3-luc cells were intraperitoneally injected into mice and the ROI was determined. (B) The ROI values represented the tumour size in each group as measured by a luciferin imaging system every 3 days. (C) Boxplots showing the tumour weight in four groups at the end of therapy. (D) The concentrations of mouse serum PAF were determined in each of the four groups before and after treatment with the different drugstore combinations. (E) Protein expression of PAFR in ovarian cancer tissues of four groups by immunohistochemistry (IHC). After 3 weeks of CDDP treatment, the PAFR expression was increased significantly (IHC, $10 \times,{ }^{\star} P>0.05$, $\left.{ }^{\star \star} P<0.05,{ }^{*} \star * P<0.01\right)$. CDDP, CDDP treatment group; DMSO, DMSO treatment group; $\mathrm{GB}, \mathrm{GB}$ treatment group; GB $+\mathrm{CDDP}, \mathrm{GB}+\mathrm{CDDP}$ treatment group.

marked increase in PI3K/ERK phosphorylation in the ovarian cancer cells exposed to CDDP and PAF. We also observed that the LY294002 and PD98059 treatments sensitised ovarian cancer cells to CDDP. The findings suggest that induction of PAFR by CDDP may lead to the potentiation of the survival pathways downstream of PAFR and thus enable cancer cells to counter CDDP-induced cytotoxicity. Previously, it was shown that PAF simultaneously induced the phosphorylation of multiple proteins including EGFR. The EGFR has been described as an important regulatory effector that is capable of stimulating PI3K/ERK signalling. Thus, we speculate that PAF/PAFR may induce EGFR transactivation, thereby mediating PI3K/ERK activation. A detailed mechanism for the crosstalk between PAFR and EGFR has not yet been fully characterised.

Furthermore, in the SKOV-3-luc ovarian cancer xenograft models, we observed that the PAFR protein expression was significantly increased in the CDDP treatment group while reduced in the CDDP plus GB treatment group, suggesting that the PAFR expression is closely related to tumour progression. In addition, the concentrations of mouse serum PAF were significantly elevated in all four groups after the ovarian cancer cell injection, but the values were not altered during the treatments of the four groups. However, the PAFR-specific antagonist GB could effectively inhibit tumour growth, suggesting that when PAFR was blocked, PAF was rendered ineffective and that PAFR might have an important role in modulating the CDDP sensitivity of ovarian cancer cells.

Our findings have thus revealed a previously unrecognised role of PAFR in modulating the CDDP sensitivity of ovarian cancer cells. Our results contribute to a better understanding of the molecular mechanisms of chemotherapy responses and provide a potential target for the development of novel combination therapy.

\section{ACKNOWLEDGEMENTS}

This work was supported by National Natural Sciences Foundation of China (81202052) awarded to W Jiang.

\section{REFERENCES}

Aponte M, Jiang W, Lakkis M, Li M-J, Edwards D, Albitar L, Vitonis A, Mok SC, Cramer DW, Ye B (2008) Activation of platelet-activating factor receptor and pleiotropic effects on tyrosine phospho-EGFR/Src/FAK/ paxillin in ovarian cancer. Cancer Res 68(14): 5839-5848.

Arora S, Bhardwaj A, Srivastava SK, Singh S, McClellan S, Wang B, Singh AP (2011) Honokiol arrests cell cycle, induces apoptosis, and potentiates the cytotoxic effect of gemcitabine in human pancreatic cancer cells. PLoS One 6(6): e21573.

Baird RD, Tan DSP, Kaye SB (2010) Weekly paclitaxel in the treatment of recurrent ovarian cancer. Nat Rev Clin Oncol 7(10): 575-582.

Braeuer RR, Zigler M, Villares GJ, Dobroff AS, Bar-Eli M (2011) Transcriptional control of melanoma metastasis: The importance of the tumor microenvironment. Semin Cancer Biol 21(2): 83-88.

Bruchim I, Ben-Harim Z, Piura E, Tepper R, Fishman A (2013) Preoperative clinical and radiological features of metastatic ovarian tumors. Arch Gynecol Obstet 288(3): 615-619.

Chan SL, Mok T (2010) PARP inhibition in BRCA-mutated breast and ovarian cancers. Lancet 376(9737): 211-213.

Darst M, Al-Hassani M, Li T, Yi QF, Travers JM, Lewis DA, Travers JB (2004) Augmentation of chemotherapy-induced cytokine production by expression of the platelet-activating factor receptor in a human epithelial carcinoma cell line. J Immunol 172(10): 6330-6335.

Ferreira MA, Barcelos LS, Teixeira MM, Bakhle YS, Andrade SP (2007) Tumor growth, angiogenesis and inflammation in mice lacking receptors for platelet activating factor (PAF). Life Sci 81(3): 210-217. 
Gao A-M, Ke Z-P, Shi F, Sun G-C, Chen H (2013) Chrysin enhances sensitivity of BEL-7402/ADM cells to doxorubicin by suppressing PI3K/ Akt/Nrf2 and ERK/Nrf2 pathway. Chem Biol Interact 206(1): 100-108.

Garcia CC, Russo RC, Guabiraba R, Fagundes CT, Polidoro RB, Tavares LP, Salgado APC, Cassali GD, Sousa LP, Machado AV, Teixeira MM (2010) Platelet-activating factor receptor plays a role in lung injury and death caused by influenza a in mice. Plos Pathog 6(11): e1001171.

Ishii S, Shimizu T (2000) Platelet-activating factor (PAF) receptor and genetically engineered PAF receptor mutant mice. Prog Lipid Res 39(1): 41-82.

Jiang W, Cong Q, Wang Y-S, Ye B, Xu C-J (2012) Ginkgo may sensitize ovarian cancer cells to cisplatin: antiproliferative and apoptosis-inducing effects of ginkgolide b on ovarian cancer cells. Integr Cancer Ther 13(43): NP10-NP17.

Jiang W, Wang Y-S, Cong Q, Li M-J, Ye B, Xu C-j (2011) Effects and mechanisms of platelet-activating factor on the invasiveness of ovarian cancer cells in vitro. Zhonghua Fu Chan Ke Za Zhi 46(12): 931-935.

Keely S, Glover LE, Weissmueller T, MacManus CF, Fillon S, Fennimore B, Colgan SP (2010) Hypoxia-inducible factor-dependent regulation of platelet-activating factor receptor as a route for gram-positive bacterial translocation across epithelia. Mol Biol Cell 21(4): 538-546.

Kume K, Shimizu T (1997) Platelet-activating factor (PAF) induces growth stimulation, inhibition, and suppression of oncogenic transformation in NRK cells overexpressing the PAF receptor. J Biol Chem 272(36): 22898-22904.

Laffman-Johnson E (2011) Improving treatment options for ovarian cancer. Clin Pharmacol Ther 90(6): 757-757.

Li T, Southall MD, Yi QF, Pei Y, Lewis D, Al-Hassani M, Spandau D, Travers JB (2003) The epidermal platelet-activating factor receptor augments chemotherapy-induced apoptosis in human carcinoma cell lines. J Biol Chem 278(19): 16614-16621.

Liu C-J, Tsai M-M, Hung P-S, Kao S-Y, Liu T-Y, Wu K-J, Chiou S-H, Lin S-C, Chang K-W (2010) miR-31 ablates expression of the HIF regulatory factor FIH to activate the HIF pathway in head and neck carcinoma. Cancer Res 70(4): 1635-1644.

Melnikova VO, Balasubramanian K, Villares GJ, Dobroff AS, Zigler M, Wang H, Petersson F, Price JE, Schroit A, Prieto VG, Hung M-C, Bar-Eli M (2009) Crosstalk between protease-activated receptor 1 and platelet-activating factor receptor regulates melanoma cell adhesion molecule (MCAM/MUC18) expression and melanoma metastasis. J Biol Chem 284(42): 28845-28855.

Mollapour E, Linch DC, Roberts PJ (2001) Activation and priming of neutrophil nicotinamide adenine dinucleotide phosphate oxidase and phospholipase $\mathrm{A}(2)$ are dissociated by inhibitors of the kinases p42(ERK2) and $\mathrm{p} 38$ (SAPK) and by methyl arachidonyl fluorophosphonate, the dual inhibitor of cytosolic and calcium-independent phospholipase A(2). Blood 97(8): 2469-2477.

Oh YS, Kim HY, Song I-C, Yun H-J, Jo D-Y, Kim S, Lee HJ (2012) Hypoxia induces CXCR4 expression and biological activity in gastric cancer cells through activation of hypoxia-inducible factor-1 alpha. Oncol Rep 28(6): 2239-2246.

Rohwer N, Dame C, Haugstetter A, Wiedenmann B, Detjen K, Schmitt CA, Cramer T (2010) Hypoxia-inducible factor 1a promotes gastric cancer chemoresistance via modulation of p53 and NF-kappa B. EJC Suppl 8(7): 97-98.

Rooth C (2013) Ovarian cancer: risk factors, treatment and management. Br J Nurs 22(17): S23-S30.

Sahu RP, Turner MJ, DaSilva SC, Rashid BM, Ocana JA, Perkins SM, Konger RL, Touloukian CE, Kaplan MH, Travers JB (2012) The environmental stressor ultraviolet B radiation inhibits murine antitumor immunity through its ability to generate platelet-activating factor agonists. Carcinogenesis 33(7): 1360-1367.

Shen D-W, Pouliot LM, Hall MD, Gottesman MM (2012) Cisplatin resistance: a cellular self-defense mechanism resulting from multiple epigenetic and genetic changes. Pharmacol Rev 64(3): 706-721.

Siddiqa A, Long LM, Li L, Marciniak RA, Kazhdan I (2008) Expression of HER-2 in MCF-7 breast cancer cells modulates anti-apoptotic proteins Survivin and Bcl-2 via the extracellular signal-related kinase (ERK) and phosphoinositide-3 kinase (PI3K) signalling pathways. BMC Cancer 8: 129.

Singh AP, Arora S, Bhardwaj A, Srivastava SK, Kadakia MP, Wang B, Grizzle WE, Owen LB, Singh S (2012) CXCL12/CXCR4 protein signaling axis induces sonic hedgehog expression in pancreatic cancer cells via extracellular regulated kinase- and akt kinase-mediated activation of nuclear factor kappa $\mathrm{b}$ implications for bidirectional tumor-stromal interactions. J Biol Chem 287(46): 39115-39124.

Ye M-X, Zhao Y-L, Li Y, Miao Q, Li Z-K, Ren X-L, Song L-Q, Yin H, Zhang J (2012) Curcumin reverses cis-platin resistance and promotes human lung adenocarcinoma A549/DDP cell apoptosis through HIF-1 alpha and caspase-3 mechanisms. Phytomedicine 19(8-9): 779-787.

Yoon H, Min J-K, Lee JW, Kim D-G, Hong HJ (2011) Acquisition of chemoresistance in intrahepatic cholangiocarcinoma cells by activation of AKT and extracellular signal-regulated kinase (ERK)1/2. Biochem Biophys Res Commun 405(3): 333-337.

Yu S-M, Kim S-J (2013) Thymoquinone-induced reactive oxygen species causes apoptosis of chondrocytes via PI3K/Akt and p38kinase pathway. Exp Biol Med 238(7): 811-820.

Zhang L, Wang D, Jiang W, Edwards D, Qiu W, Barroilhet LM, Rho J-H, Jin L, Seethappan V, Vitonis A, Wang J, Mok SC, Crum C, Cramer DW, Ye B (2010) Activated networking of platelet activating factor receptor and FAK/STAT1 induces malignant potential in BRCA1-mutant at-risk ovarian epithelium. Reprod Biol Endocrino 8: 74.

This work is published under the standard license to publish agreement. After 12 months the work will become freely available and the license terms will switch to a Creative Commons AttributionNonCommercial-Share Alike 3.0 Unported License. 